\title{
RAPID PHOTOMETRIC AND SPECTROSCOPIC VARIATIONS OF THE X-RAY SOURCE CYG X-2
}

\author{
Jerome Kristian,* Allan Sandage, and J. A. Westphal \\ Mount Wilson and Palomar Observatories, Carnegie Institution of Washington, \\ California Institute of Technology \\ Received October 3, 1967
}

\begin{abstract}
Fluctuations of $0.04 \mathrm{mag}$ on a time scale of minutes have been found in the optical flux of the candidate star for Cyg X-2. Comparison of radial velocities on a well-widened spectrogram with those of Lynds shows large changes, confirming an earlier suspicion of binary motion by $\mathbf{E}$. M. Burbidge. The systemic velocity is $\gamma=-250 \mathrm{~km} / \mathrm{sec}$. A lower limit of $300 \mathrm{~km} / \mathrm{sec}$ for the orbital velocity of one component relative to the other is indicated. The system parameters are $m_{1} / m_{2}=1, P<0.4 m \odot$ (days), and $a<0.01 m \odot$ a.u., indicative of a very close binary system $\left(m=m_{1}+m_{2}\right)$. Eclipses are probable and may have been observed. Spectroscopic evidence may be present for gas streams moving with speeds of greater than $700 \mathrm{~km} / \mathrm{sec}$. Three independent arguments are given why the optical flux cannot primarily be non-thermal radiation connected with the production of the $\mathrm{X}$-rays.
\end{abstract}

A proposed identification of optical Cyg X-2 has recently been given by Giacconi, Gorenstein, Gursky, Usher, Waters, Sandage, Osmer, and Peach (1967), based on (1) an abnormal ultraviolet intensity exhibited on a three-color photograph, and (2) an observed optical variation on a time scale of several weeks. A spectrogram of the candidate by Lynds (1967) on June 9, 1967, gave considerable credence to the identification because of the spectral peculiarities of $\mathrm{He} \Pi \lambda 4686$ in emission and a very broad $\mathrm{H} \beta$ line.

We report here new observations of optical variability on a time scale of several minutes and new spectrographic data which establish that the object is a binary. Similar results on the binary nature have been obtained by Burbidge, Lynds, and Stockton (1967), and we, in fact, were alerted to the possibility of variations in radial velocity by E. M. Burbidge in late July.

Broad-band $U B V$ photometric data were obtained for Cyg X-2 on seven nights with the 200-inch Hale reflector. These data, given in Table 1, show that variations of 0.3 mag occur between successive nights, and further that the observed magnitudes are brighter than an extrapolated bremsstrahlung spectrum fitted to the $\mathrm{X}$-ray data. The prediction using the theoretical frequency dependence of bremsstrahlung, gave $V=15.1$, $B-V=+0.1$, and $U-B=-0.9$ if no absorption and reddening is present in the field, or $V=16.1, B-V=0.4, U-B=-0.7$ if an absorption of 1 mag with normal reddening is assumed (Giacconi et al. 1967). The observations of Table 1 do not agree with these predictions in either color or magnitude, with or without absorption. This disagreement, together with more powerful evidence now to be discussed on the very rapid light fluctuations, and on the central depths of the observed absorption lines in the optical spectrum show conclusively that most of the optical light of the system is not from the free-free continuum of the hot plasma responsible for the $\mathrm{X}$-rays.

Our unpublished observations of Sco X-1, obtained between April and June, 1967, show that the optical flux of this source varies continuously with an amplitude of about 5 per cent $(0.05 \mathrm{mag})$ on a time scale of several minutes. A similar phenomenon was looked for in the Cyg X-2 candidate on the supposition that if found, strong additional evidence would be available for a positive identification. We monitored the optical flux of the candidate in the wavelength interval $\lambda \lambda 3200-8000 \AA$ for about 5 hours on July 31/August 1 (P.S.T.) at the cassegrain focus of the 100 -inch reflector using a special

* On leave of absence from the University of Wisconsin. 
double-beam ratio photometer (McCord 1967). With this device, in-focus images of two stars are alternately passed through the same focal-plane diaphragm to a single photomultiplier at a 30-hertz rate by means of a special chopper. The optical arrangement permits two stars of any relative orientation to be measured as long as their linear separation in the focal plane of the telescope is less than $70 \mathrm{~mm}$ and greater than $1.5 \mathrm{~mm}$. The individual photon pulses from each star are amplified, sorted, and recorded by two counters, one for each star, for $12.5 \mathrm{sec}$ of each $15-\mathrm{sec}$ interval. The total counts are punched on cards by an on-line key punch. Sky readings are subtracted and the intensity ratios are obtained for each 15-sec period by the Caltech IBM 7094 computer.

During these measurements, no attempt was made to normalize the response through each optical channel, so although the ratios are not, in fact, the true intensity ratios between Cyg X-2 and the comparison star, differing from the true ratio by a factor close to, but not exactly equal to 1 , the change in the ratio is accurate and represents a variation of the optical flux of one of the stars. Variations of seeing, transparency, and extinction are, of course, compensated by the system, and no extinction corrections are necessary.

TABLE 1

PHOTOMETRY OF CYG X-2

\begin{tabular}{|c|c|c|c|}
\hline Date & $V$ & $B-V$ & $U-B$ \\
\hline $\begin{array}{l}\text { April } 12 / 13,1967 \ldots \ldots \ldots \\
\text { June } 10 / 11,1967 \ldots \ldots \ldots \\
\text { Aug. } 12 / 13,1967 \ldots \ldots \ldots \ldots \\
\text { Aug. } 13 / 14,1967 \ldots \ldots \ldots \\
\text { Sept. } 10 / 11,1967 \ldots \ldots \ldots \ldots \\
\text { Sept. } 11 / 12,1967 \ldots \ldots \ldots \ldots \\
\text { Sept. } 12 / 13,1967 \ldots \ldots \ldots \ldots\end{array}$ & 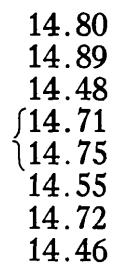 & $\begin{array}{r}0.50 \\
.50 \\
.40 \\
.43 \\
.43 \\
.45 \\
.44 \\
0.39\end{array}$ & $\begin{array}{l}-0.04 \\
-\quad .01 \\
-.40 \\
-.21 \\
-.26 \\
-.29 \\
-.27 \\
-0.36\end{array}$ \\
\hline
\end{tabular}

The results of $4 \frac{3}{4}$ hours of continuous monitoring are summarized in Figure 1 . The comparison star used in forming the relative intensity ratio is star 11 in Figure 3 of Giacconi et al. (1967), also identified on Figure 2 of the same reference at $6 \mathrm{~mm}$ south and $1 \mathrm{~mm}$ east of the Cyg X-2 candidate. The number of data points represented in Figure 1 is large. Four data dumps per minute for $4 \frac{3}{4}$ hours gave 1146 individual samples of the intensity ratio. The probable error per sample is +0.008 mag. The original data have been smoothed in the computer with a Gaussian "filter" of equivalent 2-min half width. The photon statistics of the resulting smoothed plot of Figure 1 is equivalent to \pm 0.004 mag which is indicated in the diagram as $\sqrt{ } N$. The diagram has been drawn by computer and is the filtered digital record.

Most of the fluctuations seen in Figure 1 are real and are not a result of statistical noise, as shown by the smallness of the error box. Several components of the fluctuation are visible by inspection. There are relatively short, peaked oscillations of amplitude about $0.04 \mathrm{mag}$ with rise and fall times of the order of $3 \mathrm{~min}$, separated by about 11 min. These are superposed on a longer term fluctuation of about 1 hour period, of amplitude $0.10 \mathrm{mag}$, superposed in turn on a gradual decline of about 15 per cent over the 5-hour interval. A computed Fourier power spectrum confirms these visual impressions and shows other components as well. The spectrum is definitely non-white.

The general appearance of Figure 1 is very similar to data we have obtained on Sco $\mathrm{X}-1$ during parts of seven separate nights. In particular short period components $(P \sim$ minutes $)$ are present in both objects as shown by Fourier power spectrum plots which reveal that significant energy resides in frequency components of $4 \mathrm{~min}$ or less, 
as detected by Lawrence, Ostriker, and Hesser (1967), and by us, superposed on a longer period fluctuations of the order of an hour (Mook 1967; Westphal, Sandage, and Kristian 1968). Because Sco X-1 is positively identified, the similar and highly unusual variations of the Cyg X-2 candidate are good evidence for its identification as optical Cyg X-2.

In our opinion, the presence of semi-periodic fluctuations as short as several minutes rules out the possibility that optical bremsstrahlung is the source of most of the visible light of the system. Nearly coherent, non-random fluctuations of either the electron density or the temperature would be required in the entire plasma cloud in time scales of the order of $200 \mathrm{sec}$. A more plausible possibility is the pulsation of one of the stellar components of the system, similar to the known phenomena of DQ Her (Walker 1961),

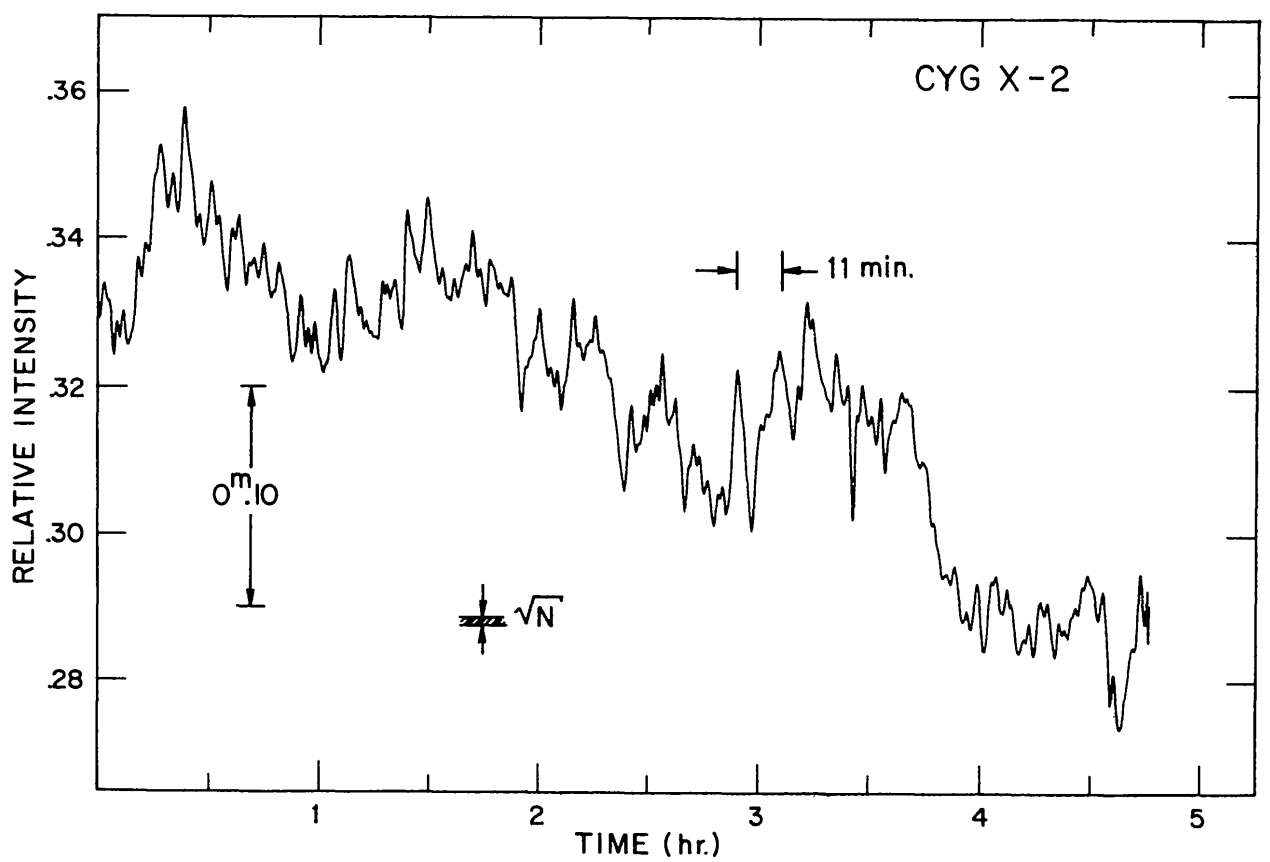

Fig. 1.-Computer-drawn intensity ratio of Cyg X-2 to a nearby comparison star as a function of time. The band pass of the photometer was $\lambda 3200$ to $\lambda 8000 \AA$. The observations were made during the night of July 31/Aug. 1, 1967 (P.S.T.), with the Mount Wilson 100-inch reflector. The uncertainty due to photon statistics is shown as an error box.

in which case we observe primarily the thermal radiation from the stellar surface in optical wavelengths. This does not, of course, rule out the possibility of a minor contamination of the total optical radiation by a bremsstrahlung source, but this source clearly cannot dominate the visible field.

Additional evidence that the contamination from any non-thermal source is small in optical wavelengths comes from a study of the line depths of absorption features in the optical spectrum. Figure 2 (Plate L1) is a reproduction of a spectrogram taken on July 30/31, 1967, with the prime-focus spectrograph of the 200-inch telescope at a dispersion of $190 \AA / \mathrm{mm}$. The resolution of this spectrogram is about $4 \AA$, which results from the projected slit width of $20 \mu$ on a IIaO baked plate at the stated dispersion. Among the remarkable features of this spectrum are the very broad hydrogen lines in absorption, each with a notable absence of damping wings such as are present in white dwarfs. Lynds (1967) noted the extreme width of $\mathrm{H} \beta$ on his spectrogram of June 9, 1967, and a width of 10-20 $\AA$ for $\mathrm{H} \gamma$ and $\mathrm{H} \delta$.

We have been able to obtain approximate intensity profiles of $\mathrm{H} \gamma$ and $\mathrm{H} \delta$ by microphotometry of the plate from which Figure 2 (Plate L1) was reproduced, using a simul- 


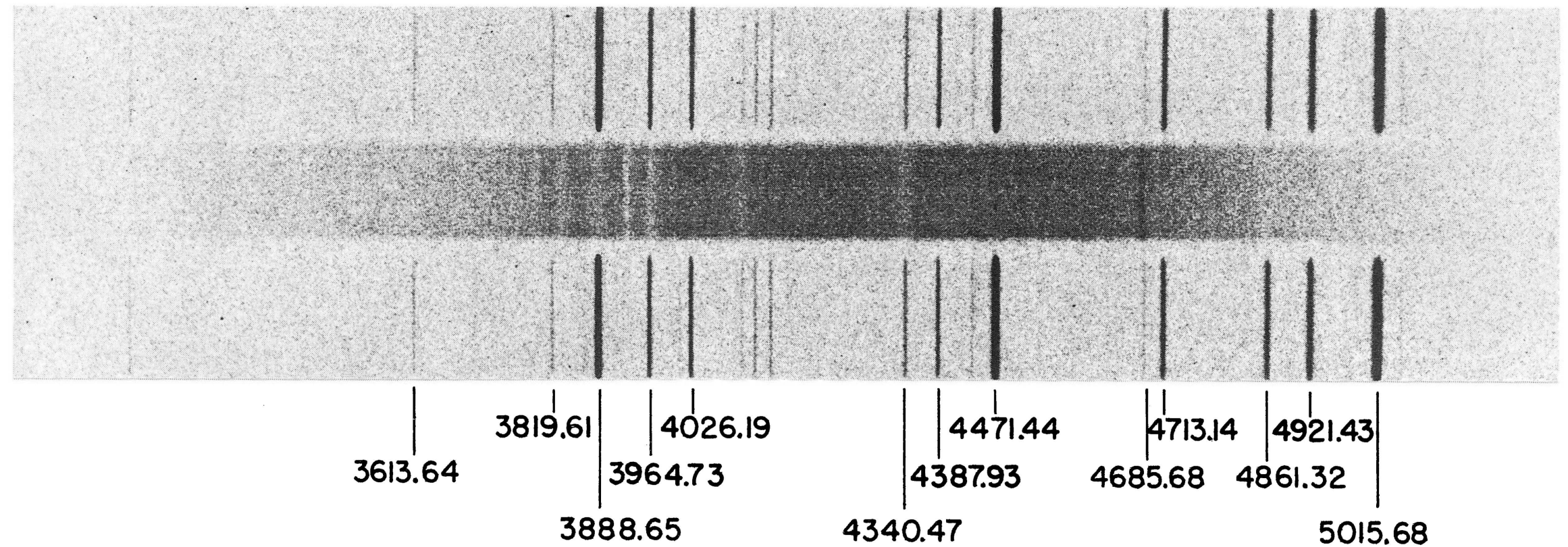

FIG. 2.-Spectrum taken July 30/31, 1967, at the prime focus of the Hale 200-inch reflector. Wavelengths of the helium comparison are marked. Original dispersion was $190 \AA / \mathrm{mm}$. The projected slit width is $3.8 \AA$.

KRISTIAN et al. (see page L101) 
taneously exposed and developed wedge made with the calibration spectrograph at Palomar. The plate response obtained with a photoelectric microphotometer was transmitted by underground cable to the California Institute of Technology computing center, where the signal was automatically sampled every $3.8 \mu(0.72 \AA)$ and recorded on magnetic tape by a high-speed analogue-to-digital converter. The wedge was measured similarly, and the reduction to intensity done by computer.

Figure 3 shows a tracing of Figure 2 in relative intensity units at a given wavelength. No absolute calibration as a function of wavelength exists, so the spectrum at different wavelengths cannot be interrelated. This is, of course, the usual case in photographic microphotometry. The principal features to note in Figure 3 are: (1) the narrowness of the $\mathrm{He}$ II line $\lambda 4686$ in emission and the $\mathrm{Ca}$ II K-line in absorption; (2) the clearly visible abnormal widths of $\mathrm{H} \gamma, \mathrm{H} \delta, \mathrm{H} 8, \mathrm{H} 9$, and $\mathrm{H} 10$; (3) the strong asymmetry with

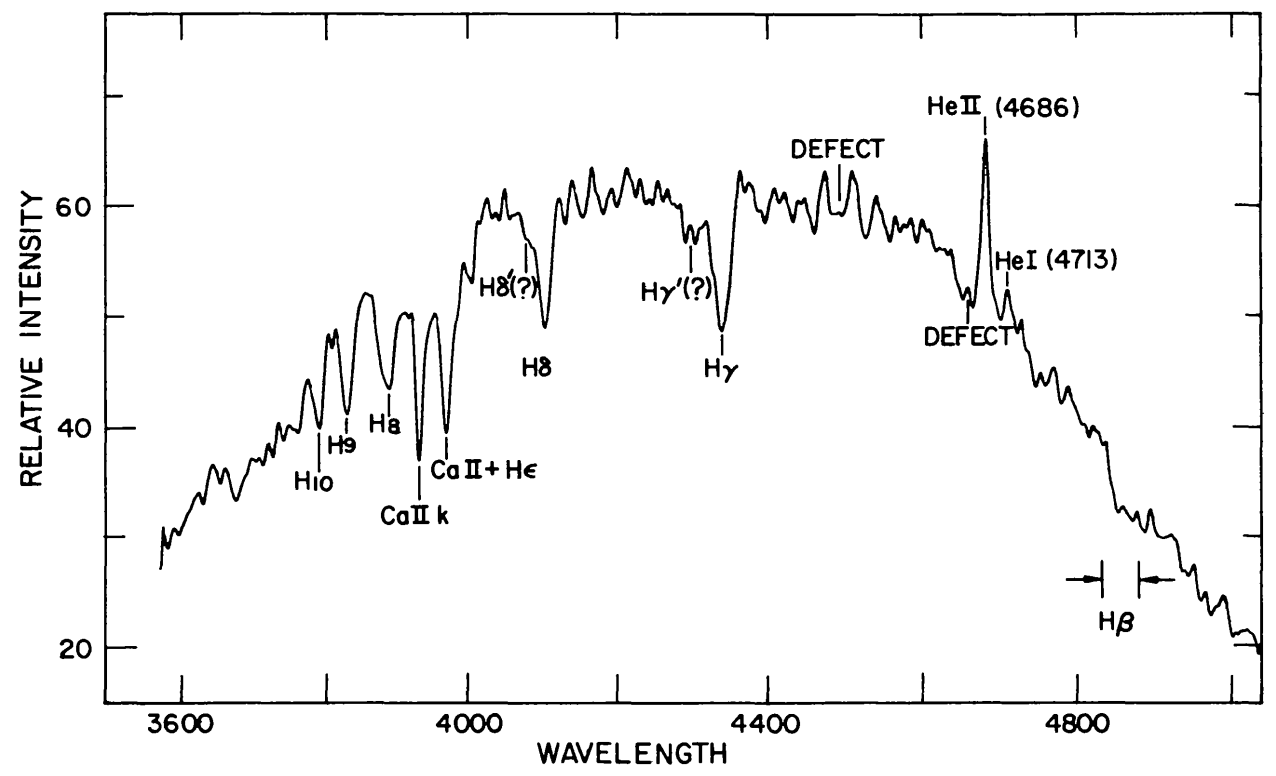

FIG. 3.-Computer-drawn rectified microphotometer tracing of the plate shown in Fig. 2 (Plate L1) smoothed with a $7 \AA$ Gaussian filter. Relative intensities apply at a given wavelength. The plate sensitivity as a function of wavelength has not been removed.

its accompanying shallow absorption immediately blueward of $\mathrm{H} \gamma$ and $\mathrm{H} \delta$ (a similar suggested absorption blueward of emission He II $\lambda 4686$ ) is a broad plate defect visible as a half-moon shaped smaller density region in Figure 2 (Plate L1); (4) a suggested absorption feature at $\lambda 4460$ which is clearly visible as a real line on the plate; and (5) a just visible indication of $\mathrm{H} \beta$ near the red-sensitivity cut-off of the IIaO plate. A further important feature, visible on the plate and marginally present in Figure 3 , is the faint emission line at $\lambda 4710$, undoubtedly due to He I at $\lambda_{0}=4713.1 \AA$. The presence of both He I $\lambda 4713$ and He II $\lambda 4686$ in the same spectrum is very rare (Greenstein, private communication). This, together with (1) the abnormal appearance of the hydrogen lines as broad, shallow, with no damping wings, and (2) the shell-like appearance of the $\mathrm{Ca}$ II lines marks the spectrum as one of the most unusual known, and makes the identification with Cyg X-2 highly probable.

The central depth of at least $A_{c} \approx 0.4$ for the sharp Ca II K-shell line, and the appreciable depths of the hydrogen lines, is additional evidence that the optical flux is not dominated by a non-thermal component such as bremsstrahlung. The data on line depths do not permit as much as half the energy to be non-thermal. Closer limits are not possible because the hydrogen lines themselves, with their absence of damping wings, 
are so abnormal in shape that comparison with normal spectra is meaningless. However, an upper limit of less than one-half the flux in non-thermal form appears to be firm. In fact, it should be emphasized that there is no evidence that any of the flux is non-thermal.

The width of the hydrogen lines and their lack of damping wings is of considerable interest. Figure 3 shows that the red side of $\mathrm{H} \gamma$ and $\mathrm{H} \delta$ rise abruptly to the continuum, and although the placement of the continuum level is uncertain by perhaps 3 per cent, this is no evidence for the presence of wings of any kind. Figure 4 illustrates this strange behavior by comparing our symmetrized $\mathrm{H} \gamma$ and $\mathrm{H} \delta$ profiles with those of the white dwarf $\mathrm{BD}+28^{\circ} 4211$ tabulated by Greenstein (1960). The profile of Cyg X-2 clearly contains no Stark-broadened component.

The total width at half-intensity is $26 \AA$ for $\mathrm{H} \gamma$, and $20 \AA$ for $\mathrm{H} \delta$. The absence of damping wings suggests that this width might be all Doppler motion, in which case

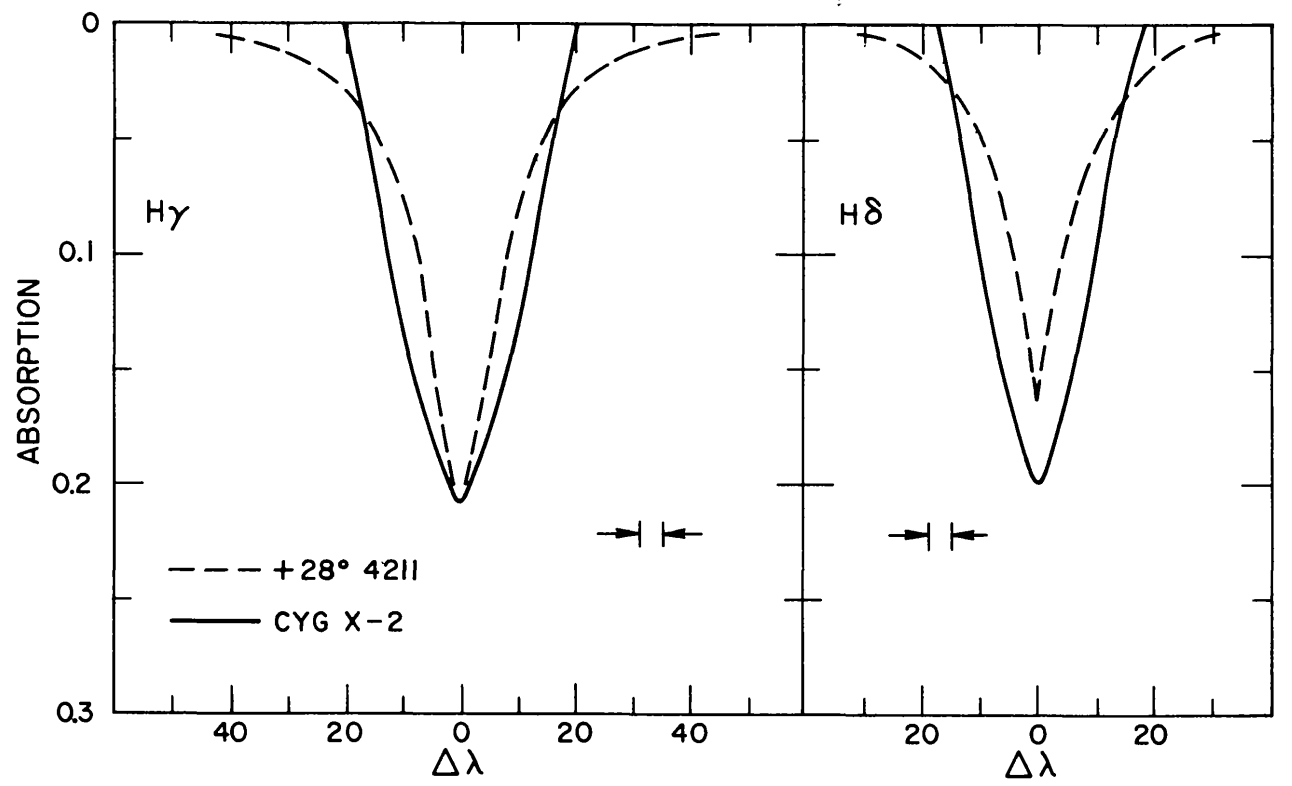

Fig. 4.-Comparison of the line profiles in $\mathrm{H}_{\gamma}$ and $\mathrm{H} \delta$ of $\mathrm{Cyg} \mathrm{X}-2$ and the white dwarf $\mathrm{BD}+28^{\circ} 4211$. The absence of damping wings is evident for the $\mathrm{X}$-ray star. The projected slit width of $3.8 \AA$ is indicated.

speeds in excess of $\pm 900 \mathrm{~km} / \mathrm{sec}$ are present. Other evidence for such velocities of gaseous streams within the system may be available from the asymmetry and apparent displaced components of $\mathrm{H} \gamma$ and $\mathrm{H} \delta$ toward the blue. These features are shown most clearly in Figure 3 and are marked $\mathrm{H} \gamma^{\prime}\left(\right.$ ?) and $\mathrm{H} \delta^{\prime}($ ?). The displacements of the asymmetries on the blue side and of the discrete absorptions range from about 10-25 $\AA$ blueward of the line centers. The definite absorption line at $\lambda 4460 \AA$, if interpreted as displaced He II at $\lambda_{0}=4471 \AA$, is shifted blueward by $11 \AA$. These shifts correspond to velocities in the range of about $1000 \mathrm{~km} / \mathrm{sec}$, and because of the discrete nature of the absorptions at $4460 \AA$ and $\mathrm{H} \gamma^{\prime}($ ?) detached gas streams are suggested, as in close binary systems like DQ Her, UX UMa, and others.

It is, therefore, of interest that Cyg X-2 is in fact a spectroscopic binary of short period. This has now been conclusively shown by Burbidge et al. (1967), and independently by our data from the spectrogram of Figure 2 (Plate L1).

The digitized data from the microphotometer scans were filtered with an $18 \AA$ Gaussian filter and the centers of the resulting lines located, as the positions of zero slope, to $0.1 \AA$. The plate was also measured independently in the usual way on a measuring machine. The results are consistent, although the accuracy of the microphotometer determinations 
is higher. It is, at any rate, limited by the accuracy of the plate itself, which introduces uncertainties of the order of $1 \AA$ or $75 \mathrm{~km} / \mathrm{sec}$. The uncertainties of the fainter, broader features are of the order of $100 \mathrm{~km} / \mathrm{sec}$. Table 2 gives measured radial velocities for the $\mathrm{He}$ emission line and for a number of identified absorption lines. The last entry in this table is the very faint absorption line measured at $\lambda 4460 \AA$ and tentatively identified as He $\lambda 4471$. With the exception of this line, and to within the stated uncertainty, all of the lines in Table 2 show the same radial velocity of the order of $-250 \mathrm{~km} / \mathrm{sec}$. But Lynds's (1967) spectrum of June 9 showed velocities of the order of $-100 \mathrm{~km} / \mathrm{sec}$ for the $\mathrm{He}$ II emission and $-400 \mathrm{~km} / \mathrm{sec}$ for the absorption lines, and a spectrum taken on July 2 by E. M. Burbidge (Burbidge et al. 1967) showed these numbers to be almost exactly reversed. It is clear, then, that the lines of the spectrum shift absolutely and with respect to each other, the absorption lines moving opposite to the He II emission. On the evidence it appears that the system is a binary in which the region producing the $\mathrm{He}$ II line moves with one of the components, and the region producing the absorption lines, including the $\mathrm{Ca}$ II shell lines, moves with the other.

By assuming circular orbits, the observations of Lynds and of Mrs. Burbidge can be used to find the velocity of the center of mass, the mass ratio of the binary pair, and upper limits for the separation and period. If $V_{\mathrm{H}}$ is the speed in orbit of the object associated

TABLE 2

MEASURED RADIAL VELOCITIES OF IDENTIFIED LINES OF THE SPECTRUM SHOWN IN FIGURES 2 AND 3

\begin{tabular}{|c|c|c|c|c|c|c|c|c|c|c|}
\hline Line & $\begin{array}{c}\mathrm{He} \mathrm{I} \\
\lambda 4713 \\
\mathrm{em}\end{array}$ & $\begin{array}{c}\text { He II } \\
\lambda 4686 \\
\text { em }\end{array}$ & $\begin{array}{c}\mathrm{Ca} \text { II } \\
\mathbf{K}\end{array}$ & $\mathrm{H}_{\gamma}$ & $\mathrm{H} \delta$ & $\begin{array}{c}\mathrm{H} \epsilon+ \\
\mathrm{Ca} \text { IIH }\end{array}$ & $\begin{array}{c}\mathrm{H} 8 \\
(+\mathrm{He} \\
\mathrm{I} ?)\end{array}$ & H9 & $\mathrm{H} 10$ & $\begin{array}{c}\mathrm{He} \\
\lambda 4471 \\
(?)\end{array}$ \\
\hline Radial velocity $(\mathrm{km} / \mathrm{sec})$ & -250 & -250 & -300 & -230 & -130 & -230 & -180 & -230 & -370 & $-80 c$ \\
\hline
\end{tabular}

with the absorption lines, then the measured radial velocity at time $t$ will be $V_{R}$ (measured) $=\gamma+V_{\mathrm{H}} f(t)$, where $f(t)$ includes the phase and the inclination of the orbit. A similar equation, with a negative sign for the time-dependent term, will hold for the He II line. By solving these equations, using the measured values from the two spectra, we obtain $\gamma \approx-250 \mathrm{~km} / \mathrm{sec}$ and $V_{\mathrm{He}} / V_{\mathrm{H}} \approx 1$, which means that the masses of the components are approximately equal. In both cases $|V f(t)| \approx 150 \mathrm{~km} / \mathrm{sec}$, and since $|f(t)| \leq 1$, this gives a lower limit for $V$ of $150 \mathrm{~km} / \mathrm{sec}$, or for the relative speed of the binary components about $300 \mathrm{~km} / \mathrm{sec}$. This in turn gives upper limits of about 0.01 $m$ (a.u.) for the separation, and $0.4 m$ days for the period, where $m$ is the mass of the system $\left(m_{1}+m_{2}\right)$ in solar units. It should be emphasized that these limits are good only to within the uncertainties of the velocity determination. If the velocities in orbit are in error by a factor of 2 , which seems possible, then the limits stated for the separation and period will be in error by factors of 4 and 8 , respectively. On the other hand, confidence in the estimates is increased by the supporting evidence of our spectrum, which was taken near conjunction. For an earlier discussion of similar calculations applied to double lined binaries see Wilson (1941).

It seems then, from the minimal data now available, that the system is a binary of very short period, and may even be a contact binary. There is also some indication from the photometric data that the system may be an eclipser, which would be consistent with the large radial velocities observed, which imply a large orbital inclination. The $U B V$ photometry given in Table 1 shows that the changes of color from night to night are correlated with the changes of magnitude, the system becoming redder when it becomes fainter. It is also possible that the gradual decline of $0.15 \mathrm{mag}$ in intensity over 
the 5 hours of continuous observation shown in Figure 2 is due to an eclipse. With this interpretation, the time scale of the decline would be consistent with the limit previously assigned to the period from the spectroscopic data. It is hoped that further observations will soon decide the question.

A final point of some interest concerns the population type of Cyg X-2. The galactic coordinates are $l^{\mathrm{II}}=87^{\circ}$ and $b^{\mathrm{II}}=-11^{\circ}$, so that the object lies nearly in the local direction of galactic rotation. The measured systematic velocity of $-250 \mathrm{~km} / \mathrm{sec}$ is therefore due almost entirely to the Sun's circular motion of $250 \mathrm{~km} / \mathrm{sec}$. The rotational motion of the object with respect to the Galaxy must then be quite small, and the eccentricity of its galactic orbit quite large. On this evidence, Cyg X-2 must be classified as a Population II object, an interesting point in view of recent discussions of the possible association of X-ray sources with young Population I objects (O'Dell 1967; Braes and Hovenier 1966).

We are greatly indebted to E. M. Burbidge for informing us of her results at an early date, to J. L. Greenstein for a very illuminating discussion, to Tom McCord for graciously lending his photometer to us, and to E. W. Dennison and his group, without whose data-system electronics the 100 -inch photometry could not have been done. Kristian's work was supported in part by grants from the National Science Foundation and the Office of Naval Research.

\section{REFERENCES}

Braes, L. L. E., and Hovenier, J. W. 1966, Nature, 209, 360.

Burbidge, E. M., Lynds, C. R., and Stockton, A. 1967, A p. J. (Letters), 150, L95.

Giacconi, R., Gorenstein, P., Gursky, H., Usher, P. D., Waters, J. R., Sandage, A., Osmer, P., and Peach, J. V. 1967, Ap.J. (Letters), 148, L129.

Greenstein, J. L. 1960, "Spectra of Stars below the Main Sequence," in Stars and Stellar Systems, Vol. 6, Stellar Atmospheres, ed. J. L. Greenstein (Chicago: University of Chicago Press), p. 676.

Lawrence, G. M., Ostriker, J. P., and Hesser, J. E. 1967, Ap. J. (Letters), 148, L161.

Lynds, C. R. 1967, Ap. J. (Letters), 149, L41.

McCord, T. 1967, "A Double Beam Astronomical Photometer," submitted to Applied Optics.

Mook, D. E. 1967, presented at Yerkes Meeting of A.A.S., June, 1967.

O'Dell, C. R. 1967, $A$ p.J., 147, 855.

Walker, M. F. 1961, Ap.J., 134, 171.

Westphal, J. A., Sandage, A., and Kristian, J. 1968, Ap. J. (in preparation).

Wilson, O. C. 1941, Ap.J., 93, 29. 